\title{
A Comparison of Narcotic Consumption Between Hospital and Ambulatory-Based Surgery Centers Following Anterior Cervical Discectomy and Fusion
}

\author{
DUSTIN H. MASSEL, MD, ANKUR S. NARAIN, MD, FADY Y. HIJJI, MD, BENJAMIN C. MAYO, MD, \\ DANIEL D. BOHL, MD, MPH, GREGORY D. LOPEZ, MD, KERN SINGH, MD \\ Department of Orthopaedic Surgery, Rush University Medical Center, Chicago, Illinois
}

\begin{abstract}
Background: Several studies have compared outcomes between hospital-based (HBCs) and ambulatory surgery centers (ASCs) following anterior cervical discectomy and fusion (ACDF). However, the association between narcotic consumption and pain in the early postoperative period has not been well characterized. As such, the purpose of this study is to compare pain, narcotic consumption, and length of stay (LOS) between HBC and ASC patients undergoing same-day-discharge following ACDF.

Methods: A surgical registry of patients who underwent a primary, 1- or 2-level ACDF during 2013-2015 was reviewed. Patients were stratified by operative location. Differences in demographics were assessed using independentsample $t$ tests and chi-square analysis. The presence of an association between operative location and outcomes was analyzed using Poisson regression with robust error variance or linear regression adjusted for preoperative characteristics.

Results: A total of 76 patients were identified, of which 42 and 34 underwent surgery at an HBC or ASC, respectively. The HBC cohort had greater total $(P<.001)$ and hourly $(P=.034)$ narcotic consumption and prolonged LOS $(P<.001)$. Over $90 \%$ of ASC patients consumed less than or equal to the 30 th percentile $(32.0 \mathrm{mg})$ of oral morphine equivalents (OME), whereas over $57 \%$ of HBC patients consumed greater than $32.0 \mathrm{mg}$ OME. The HBC cohort consumed greater average doses of fentanyl and oxycodone $(P<.001$ for each).

Conclusions: This study demonstrates that patients undergoing same-day surgery for primary 1- or 2-level ACDF received more narcotics at $\mathrm{HBCs}$ compared to at ASCs. The increased narcotic consumption at HBCs may have resulted in longer LOS; however, this did not impact long-term pain, complications, or clinical outcomes.

Clinical Relevance: Patients scheduled to be discharged on postoperative day 0 following ACDF at HBCs may be able to receive fewer narcotics and be discharged sooner without compromising pain control or increasing their risk for complications.
\end{abstract}

Cervical Spine

Keywords: anterior cervical discectomy and fusion, narcotic consumption, inpatient pain, same-day discharge, hospital, ambulatory surgery center

\section{INTRODUCTION}

Degenerative spinal pathology directly contributes to health care expenditure through diagnostic testing and treatment costs and indirectly through disability expenses. ${ }^{1-3}$ Following low back pain, neck pain is the second most common musculoskeletal complaint in the primary care setting and has contributed to a $15 \%$ increase in ambulatory visits in recent years. ${ }^{4-6}$ Advancements in spinal instrumentation and ambulatory surgery facilities, in conjunction with the growing demand for low-cost, high-efficacy treatment options, has resulted in a dramatic increase in outpatient surgical management. ${ }^{7}$

Anterior cervical discectomy and fusion (ACDF) is one of the most commonly performed spinal surgery procedures in both the inpatient and the outpatient setting. ${ }^{8}$ Several studies have demonstrated the safety and efficacy of ACDF performed as an outpatient procedure. ${ }^{7,9-14}$ Compared to the inpatient setting, prior literature has reported shorter operative times and improved patient satisfaction, with similar rates of perioperative complications and successful outcomes following outpatient ACDFs. ${ }^{9-11,15}$ Although the literature 
demonstrates successful clinical outcomes, the relative impact of the surgical setting on early postoperative narcotic consumption has not been studied.

Narcotic consumption is a significant concern within the medical community due to the prevalence of narcotic prescriptions and the potential for dependence and abuse. ${ }^{16-18}$ Thus, minimizing preand postoperative narcotic requirements following ACDF is an important area to evaluate. The principal aim of the current study is to compare narcotic consumption and pain in the early postoperative period following ACDF by operative setting. The authors hypothesize that patients will consume fewer oral morphine equivalents (OMEs) on postoperative day 0 (POD 0 ) at an ambulatory surgery center (ASC) compared to a hospital-based center (HBC).

\section{MATERIALS AND METHODS}

\section{Patient Population}

Institutional review board approval was granted for this study (ORA\#14051301). A retrospective review of a prospectively collected surgical registry was performed on all patients who underwent a primary, 1- or 2-level ACDF by a single surgeon between 2013 and 2015. Patients were stratified by operative setting: HBC versus ASC. Patients were excluded from the analysis if they were discharged after postoperative day (POD) 0 . Patients with an allergy or intolerance to narcotic medications who underwent a previous spinal surgery, who had a history of spinal trauma, or who underwent ACDF with fewer than 6 months of postoperative followup were also excluded from the study.

\section{Surgical Procedure and Postoperative Analgesia Protocol}

The surgical procedure was performed identically at both the HBC and the ASC setting. All patients underwent general endotracheal anesthesia. After identification of the surgical level via fluoroscopic imaging, a 2- to $3-\mathrm{cm}$ horizontal incision was made medially to the sternocleidomastoid. The SmithRobinson approach was utilized, with the platysma being incised in line with the skin. The trachesophageal complex was retracted medially, while the carotid sheath and sternocleidomastoid were retracted laterally. After blunt dissection down to the level of the vertebral body, a fluoroscopic image was again acquired to confirm the correct surgical level.
An annulotomy was then performed, with subsequent removal of the disc material and cartilaginous end plates. The posterior longitudinal ligament was then resected. Following disc space preparation, an interbody cage packed with autograft, allograft, or bone graft substitute was introduced into the intervertebral space. Supplemental plate fixation was then performed, followed by wound irrigation and layered closure.

Postoperatively, all patients at both surgical settings received the same multimodal analgesia protocol. The protocol is detailed in Appendix A.

\section{Demographic and Outcome Analysis}

The following patient demographics and preoperative baseline characteristics were analyzed: age, sex, smoking status, body mass index (nonobese $\left[\right.$ BMI $\left.<30 \mathrm{~kg} / \mathrm{m}^{2}\right]$, obese $\left[\right.$ BMI $\left.\left.\geq 30 \mathrm{~kg} / \mathrm{m}^{2}\right]\right)$, primary insurance payer, number of operative levels, Charlson Comorbidity Index (CCI), Visual Analog Scale (VAS) for neck pain and arm pain, and Neck Disability Index (NDI). Perioperative outcome measures included operative time, estimated blood loss, length of hospital stay, and complication rates. Postoperative outcomes included average VAS neck and arm pain, average change in VAS neck and arm pain, narcotic consumption (OMEs), and complication rates.

The NDI consists of 10 questions assessing the effect of a patient's neck pain on his or her daily function. Each question is recorded from 0 to 5 , with a maximum score of 50 points indicating maximum disability.

The VAS utilizes a 10-point rating scale to assess patient-reported pain. A 0 indicates that the patient is experiencing no pain, whereas a 10 indicates that the patient is experiencing maximum pain. Postoperative VAS scores were collected identically at the HBC and ASC. VAS scores are recorded in the electronic medical record at regular intervals by the nursing staff. If multiple scores are obtained per hour, the scores are averaged to produce 1 VAS value per hour.

Postoperative narcotic consumption was assessed in the form of total, daily, and hourly OMEs. For each postoperative day, the type and dosage of each analgesic medication administered is recorded by nursing staff in the electronic medical record. This information is translated into OMEs using the formula presented in Appendix B. Distribution analysis of total OME consumption was performed. 
The 30th and 50th percentiles of OME consumption were used for the actual and theoretical analyses, respectively, in order to highlight the differences between operative settings.

\section{OME Theoretical Analysis}

To minimize the difference in length of stay (LOS) between operative settings, a theoretical analysis was performed based on when a patient was discharged from the $\mathrm{HBC}$ or the ASC. Theoretical total OME consumption was calculated by adding the predischarge OME total to a theoretical postoperative intake. The postoperative prescription at the institutions in question recommend 1 or 2 doses of $5-325 \mathrm{mg}$ hydrocodoneacetaminophen every 4 hours as needed for pain. The theoretical postoperative intake assumed that patients took 2 doses of the prescribed medication every 4 hours from their discharge time until midnight on POD 0.

\section{Statistical Analysis}

All statistical analyses were performed using Stata/MP 13.1 for Mac (StataCorp LP, College Station, Texas). Patient demographics and preoperative baseline characteristics were compared using independent-sample $t$ tests and chi-square analysis for continuous and categorical variables, respectively. The effect of operative location on VAS pain scores, NDI, and OME consumption was assessed using multivariate Poisson regression with robust error variance for binary outcomes or multivariate linear regression for continuous outcomes adjusted for preoperative characteristics, including age, sex, smoking status, BMI category, comorbidity burden, and primary insurance payer. The theoretical analysis was completed with the same statistical tests and adjusted for the same preoperative variables. A $P$ value $<.05$ was considered statistically significant.

\section{RESULTS}

\section{Primary Analysis}

A total of 76 patients were included in the analysis. Of these, $42(55.3 \%)$ underwent surgery at a HBC and $34(44.7 \%)$ at an ASC. The average patient age was $48.7 \pm 8.2$ years for the HBC cohort and $49.2 \pm$ 10.7 years for the ASC cohort $(P=.808)$. A majority of the patient population analyzed were nonobese (HBC: $66.7 \%$ versus ASC: $58.8 \%, P=.481$ ) and
Table 1. Baseline Characteristics. ${ }^{a}$

\begin{tabular}{|c|c|c|c|}
\hline & $\begin{array}{l}\text { Hospital } \\
(\mathrm{N}=42)\end{array}$ & $\begin{array}{c}\mathrm{ASC} \\
(\mathrm{N}=\mathbf{3 4})\end{array}$ & $P$ Value \\
\hline Age $($ mean $\pm \mathrm{SD}, \mathrm{y})$ & $48.7 \pm 8.2$ & $49.2 \pm 10.7$ & .808 \\
\hline $\operatorname{Sex}(n)$ & & & .684 \\
\hline Female & $42.9 \%(18)$ & $38.2 \%(13)$ & \\
\hline Male & $57.1 \%(24)$ & $61.8 \%(21)$ & \\
\hline Smoking status (n) & & & .910 \\
\hline Nonsmoker & $83.3 \%(35)$ & $82.4 \%(28)$ & \\
\hline Smoker & $16.7 \%(7)$ & $17.7 \%(6)$ & \\
\hline BMI (n) & & & .481 \\
\hline Nonobese $(\mathrm{BMI}<30)$ & $66.7 \%(28)$ & $58.8 \%(20)$ & \\
\hline Obese (BMI $\geq 30)$ & $33.3 \%(14)$ & $41.2 \%(14)$ & \\
\hline Insurance (n) & & & $<.001$ \\
\hline Workers' compensation & $14.3 \%(6)$ & $61.8 \%(21)$ & \\
\hline Private insurance & $85.7 \%(36)$ & $38.2 \%(13)$ & \\
\hline Operative levels (n) & & & .603 \\
\hline 1 level & $61.9 \%(26)$ & $67.7 \%(23)$ & \\
\hline 2 level & $38.1 \%(16)$ & $32.4 \%(11)$ & \\
\hline $\begin{array}{l}\text { Comorbidity burden } \\
\text { (CCI) }\end{array}$ & $1.4 \pm 1.4$ & $1.2 \pm 1.3$ & .566 \\
\hline $\begin{array}{l}\text { Preoperative VAS neck } \\
\text { pain }(\text { mean } \pm S D)\end{array}$ & $6.3 \pm 2.3$ & $6.8 \pm 2.0$ & .407 \\
\hline $\begin{array}{l}\text { Preoperative VAS arm } \\
\text { pain }(\text { mean } \pm \text { SD) }\end{array}$ & $5.6 \pm 2.6$ & $6.2 \pm 2.4$ & .380 \\
\hline $\begin{array}{l}\text { Preoperative NDI (mean } \\
\quad \pm \mathrm{SD} \text { ) }\end{array}$ & $40.2 \pm 16.2$ & $48.0 \pm 19.2$ & .090 \\
\hline
\end{tabular}

Abbreviations: ASC, ambulatory surgery center; SD, standard deviation; BMI, body mass index; CCI, Charlson Comorbidity Index; VAS, visual analog scale; NDI, neck disability index.

${ }^{a}$ Boldface indicates statistical significance.

male (HBC: $57.1 \%$ versus ASC: $61.8 \%, P=.684$ ). The HBC cohort had a greater percentage of patients with private insurance $(85.7 \%$ versus $61.8 \%, P<$ $.001)$ compared to the ASC cohort. The remainder of baseline characteristics are reported in Table 1.

Tables 2 and 3 detail the postoperative outcome comparisons and analysis of narcotic consumption between cohorts. The HBC cohort had longer hospital lengths of stay (LOS) $(7.4 \pm 2.7$ versus $4.7 \pm 1.2$ hours, $P<.001)$ and consumed more total OMEs $(43.2 \pm 27.0$ versus $18.2 \pm 14.1 \mathrm{mg}$ OME, $P$ $<.001)$ and hourly OMEs $(6.3 \pm 4.6$ versus $3.4 \pm$ 2.6, $P=.034$ ) compared to the ASC cohort. Figure 1 demonstrates that $90.9 \%$ of ASC patients consumed less than or equal to the 30 th percentile $(32.0 \mathrm{mg})$ of OMEs. In contrast, $57.2 \%$ of $\mathrm{HBC}$ patients consumed greater than $32.0 \mathrm{mg}$ OME. The HBC cohort consumed greater average dosages of fentanyl $(9.5 \pm 17.8$ versus $1.6 \pm 5.5 \mathrm{mg}$ OME, $P=.021)$ and oxycodone $(10.1 \pm 13.1$ versus $1.0 \pm 3.1 \mathrm{mg}$ OME, $P<.001)$. The HBC cohort also consumed greater average dosages of hydrocodone (11.5 \pm 13.3 versus $6.8 \pm 8.3 \mathrm{mg}$ OME, $P=.150)$ and tramadol $(11.2 \pm 8.3$ versus $7.6 \pm 4.4, P=.062)$; however, these differences were not statistically significant. Of the total OMEs, the HBC cohort consumed a greater percent of oxycodone $(26.6 \%$ 
Table 2. Outcomes. ${ }^{\text {a }}$

\begin{tabular}{|c|c|c|c|}
\hline & $\begin{array}{l}\text { Hospital } \\
(\mathrm{N}=42)\end{array}$ & $\begin{array}{c}\text { ASC } \\
(\mathrm{N}=34)\end{array}$ & $P$ Value $^{\mathrm{b}}$ \\
\hline Operative time (min) & $54.6 \pm 14.6$ & $57.6 \pm 14.6$ & .341 \\
\hline Estimated blood loss $(\mathrm{mL})$ & $31.1 \pm 11.8$ & $29.4 \pm 9.7$ & .204 \\
\hline Length of hospital stay (h) & $7.4 \pm 2.7$ & $4.7 \pm 1.2$ & $<.001$ \\
\hline $\begin{array}{l}\text { Inpatient VAS pain scores } \\
\text { POD } 0(\text { mean } \pm \mathrm{SD})\end{array}$ & $4.5 \pm 2.0$ & $3.9 \pm 2.1$ & .233 \\
\hline \multicolumn{4}{|l|}{ VAS neck pain (mean \pm SD) } \\
\hline 6-week VAS & $2.4 \pm 2.1$ & $4.2 \pm 2.6$ & .039 \\
\hline 12-week VAS & $2.1 \pm 2.0$ & $3.2 \pm 2.7$ & .332 \\
\hline 6-month VAS & $2.2 \pm 2.1$ & $3.7 \pm 3.2$ & .365 \\
\hline \multicolumn{4}{|c|}{ Change in VAS neck pain $(\text { mean } \pm \mathrm{SD})^{\mathrm{c}}$} \\
\hline$\Delta \mathrm{VAS}$ at 6 weeks & $-3.5 \pm 2.2$ & $-1.9 \pm 2.2$ & .024 \\
\hline$\Delta \mathrm{VAS}$ at 12 weeks & $-3.5 \pm 2.9$ & $-2.9 \pm 2.4$ & .454 \\
\hline$\Delta$ VAS at 6 months & $-3.1 \pm 2.7$ & $-1.7 \pm 2.4$ & .547 \\
\hline \multicolumn{4}{|c|}{ VAS arm pain (mean \pm SD) } \\
\hline 6-week VAS & $2.3 \pm 2.3$ & $3.1 \pm 2.4$ & .297 \\
\hline 12-week VAS & $1.7 \pm 1.9$ & $3.0 \pm 2.8$ & .194 \\
\hline 6-month VAS & $1.7 \pm 1.8$ & $3.6 \pm 3.0$ & .065 \\
\hline \multicolumn{4}{|c|}{ Change in VAS arm pain $(\text { mean } \pm \overline{\mathrm{SD}})^{\mathrm{c}}$} \\
\hline$\Delta$ VAS at 6 weeks & $-3.1 \pm 2.5$ & $-2.2 \pm 1.9$ & .064 \\
\hline$\Delta \mathrm{VAS}$ at 12 weeks & $-2.5 \pm 3.6$ & $-2.3 \pm 2.4$ & .437 \\
\hline$\Delta$ VAS at 6 months & $-2.9 \pm 3.2$ & $-2.0 \pm 2.3$ & .326 \\
\hline \multicolumn{4}{|l|}{$\mathrm{NDI}($ mean $\pm \mathrm{SD})$} \\
\hline 6-week NDI & $25.2 \pm 15.6$ & $36.8 \pm 23.0$ & .079 \\
\hline 12-week NDI & $17.1 \pm 13.3$ & $30.6 \pm 21.8$ & .097 \\
\hline & $16.1 \pm 14.2$ & $37.4 \pm 26.1$ & .072 \\
\hline \multicolumn{4}{|c|}{ Change in NDI $(\text { mean } \pm \mathrm{SD})^{\mathrm{c}}$} \\
\hline$\Delta$ NDI at 6 weeks & $-14.9 \pm 15.1$ & $-7.0 \pm 14.5$ & .064 \\
\hline$\Delta \mathrm{NDI}$ at 12 weeks & $-22.8 \pm 16.8$ & $-13.5 \pm 15.8$ & .319 \\
\hline$\Delta$ NDI at 6 months & $-23.5 \pm 16.7$ & $-12.0 \pm 23.2$ & .171 \\
\hline Complications $(\mathrm{n})^{\mathrm{d}}$ & $2.4 \%(1)$ & $0.0 \%(0)$ & - \\
\hline
\end{tabular}

Abbreviations: ASC, ambulatory surgery center; POD, postoperative day; SD, standard deviation; VAS, visual analog scale; NDI, neck disability index. ${ }^{\text {a} B o l d f a c e ~ i n d i c a t e s ~ s t a t i s t i c a l ~ s i g n i f i c a n c e . ~}$

${ }^{\mathrm{b}} P$ values calculated using Poisson regression with robust error variance controlling for age, gender, smoking status, BMI category, insurance status, Charlson Comorbidity Index, and preoperative VAS (neck and arm).

${ }^{\mathrm{c}}$ Change in VAS $=$ postoperative VAS (6 weeks, 12 weeks, or 6 months $)-$ preoperative VAS.

${ }^{\mathrm{d}}$ Urinary retention requiring recatheterization $(\mathrm{n}=1) ; P$ value not calculated due to event incidence $<5$.

versus $6.6 \%, P=.017$; Table 3, Figure 2) compared to ASCs. The ASC cohort consumed a greater percent of tramadol (54.7\% versus $29.6 \%, P=.017$; Table 3, Figure 2) compared to HBCs.

Table 3. Analysis of oral morphine equivalent (OME) consumption. ${ }^{\mathrm{a}}$

\begin{tabular}{lccr}
\hline & $\begin{array}{c}\text { Hospital } \\
(\mathbf{N}=\mathbf{4 2})\end{array}$ & $\begin{array}{c}\text { ASC } \\
(\mathbf{N}=\mathbf{3 4})\end{array}$ & $\boldsymbol{P}$ Value \\
\hline Total OME consumption & $\mathbf{4 3 . 2} \pm \mathbf{2 7 . 0}$ & $\mathbf{1 8 . 2} \pm \mathbf{1 4 . 1}$ & $<. \mathbf{0 0 1}$ \\
$\quad$ POD 0 (mean \pm SD) & & & \\
Hourly OME consumption & $\mathbf{6 . 3} \pm \mathbf{4 . 6}$ & $\mathbf{3 . 4} \pm \mathbf{2 . 6}$ & $\mathbf{. 0 3 4}$ \\
POD 0 (mean \pm SD) & & & \\
OME dose (mean \pm SD) & & & \\
Hydrocodone & $11.5 \pm 13.3$ & $6.8 \pm 8.3$ & .150 \\
Fentanyl & $\mathbf{9 . 5} \pm \mathbf{1 7 . 8}$ & $\mathbf{1 . 6} \pm \mathbf{5 . 5}$ & $\mathbf{. 0 2 1}$ \\
Tramadol & $11.2 \pm 8.3$ & $7.6 \pm 4.4$ & .062 \\
Oxycodone & $\mathbf{1 0 . 1} \pm \mathbf{1 3 . 1}$ & $\mathbf{1 . 0} \pm \mathbf{3 . 1}$ & $\mathbf{. 0 0 2}$ \\
Medications: average percent of total OMEs & & \\
Hydrocodone & $29.1 \%$ & $33.1 \%$ & .772 \\
Fentanyl & $14.2 \%$ & $5.5 \%$ & .711 \\
Tramadol & $\mathbf{2 9 . 6 \%}$ & $\mathbf{5 4 . 7 \%}$ & $\mathbf{. 0 1 7}$ \\
Oxycodone & $\mathbf{2 6 . 6 \%}$ & $\mathbf{6 . 6 \%}$ & $\mathbf{. 0 2 4}$ \\
\hline
\end{tabular}

ASC, ambulatory surgery center; SD, standard deviation; POD, postoperative day. ${ }^{a}$ Boldface indicates statistical significance.

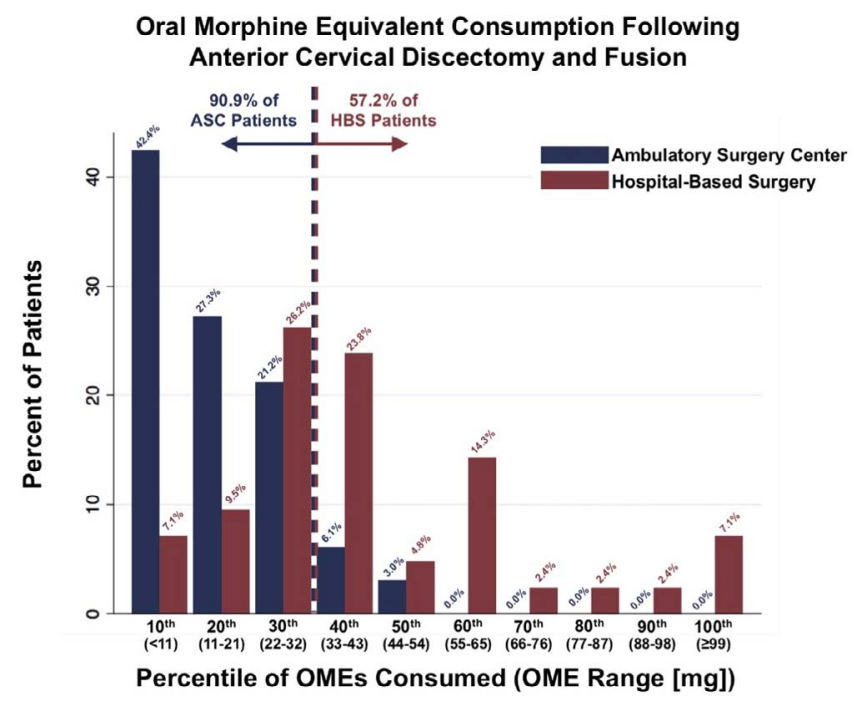

Figure 1. Oral morphine equivalent (OME) consumption following anterior cervical discectomy and fusion.

No differences were observed in VAS neck pain scores on POD 0 between cohorts (HBC: $4.5 \pm 2.0$ versus ASC: $3.9 \pm 2.1, P=.233$; Table 2 ). In contrast, the HBC cohort reported lower absolute VAS neck pain scores $(2.4 \pm 2.1$ versus $4.2 \pm 2.6, P=.039)$ and a greater improvement in VAS neck pain $(-3.5 \pm 2.2$ versus $-1.9 \pm 2.2, P=.024)$ at the 6 -week follow-up compared to the ASC cohort. This difference was not present for the remainder of the postoperative followup visits. No differences were observed in operative time, estimated blood loss, VAS neck pain after 6 weeks, VAS arm pain, change in VAS arm pain, or complication rate up to 6 months postoperatively. Complications included 1 case of urinary retention (n $=1$ ) requiring recatheterization in a patient who underwent surgery at the HBC site.

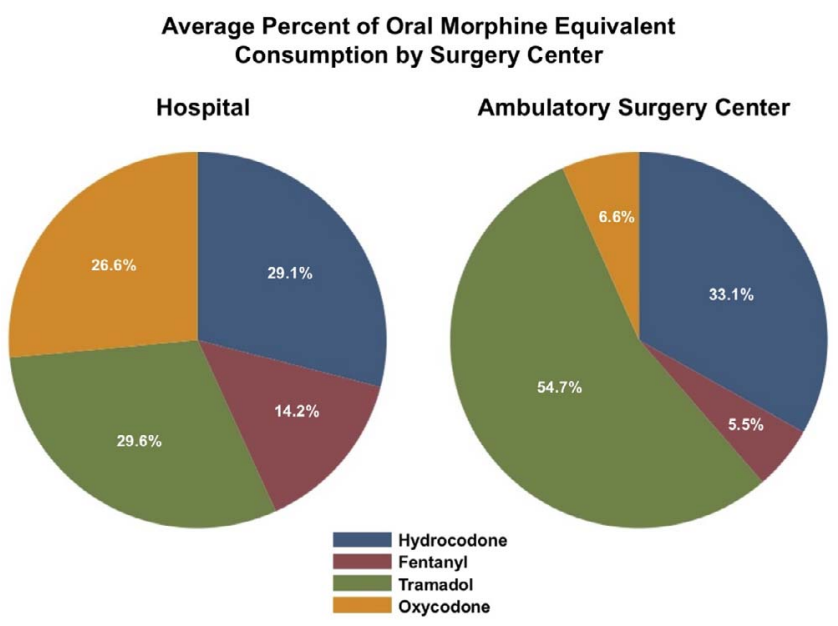

Figure 2. Average percent of oral morphine equivalent consumption by surgery center. 
Table 4. Theoretical analysis of oral morphine equivalent (OME) consumption. $^{\text {a }}$

\begin{tabular}{lccc}
\hline & $\begin{array}{c}\text { Hospital } \\
(\mathbf{N}=\mathbf{4 2})\end{array}$ & $\begin{array}{c}\text { ASC } \\
(\mathbf{N}=\mathbf{3 4})\end{array}$ & $\boldsymbol{P}$ Value \\
\hline $\begin{array}{l}\text { Total OME consumption } \\
\text { POD 0 (mean } \pm \text { SD) }\end{array}$ & $63.4 \pm 28.8$ & $50.3 \pm 12.7$ & .397 \\
Hourly OME consumption & $5.1 \pm 2.5$ & $3.7 \pm 0.9$ & .147 \\
POD 0 (mean \pm SD) & & & \\
OME dose (mean \pm SD) & & & \\
Hydrocodone & $\mathbf{3 1 . 7} \pm \mathbf{1 4 . 9}$ & $\mathbf{4 0 . 2} \pm \mathbf{1 0 . 4}$ & $\mathbf{. 0 4 4}$ \\
Fentanyl & $\mathbf{9 . 5} \pm \mathbf{1 7 . 8}$ & $\mathbf{1 . 6} \pm \mathbf{5 . 5}$ & $\mathbf{. 0 2 1}$ \\
Tramadol & $11.2 \pm 8.3$ & $7.6 \pm 4.4$ & .062 \\
Oxycodone & $\mathbf{1 0 . 1} \pm \mathbf{1 3 . 1}$ & $\mathbf{1 . 0} \pm \mathbf{3 . 1}$ & $\mathbf{. 0 0 2}$ \\
Medications: average percent of total OMEs & & \\
Hydrocodone & $\mathbf{5 5 . 7 \%}$ & $\mathbf{8 0 . 7 \%}$ & $\mathbf{. 0 1 4}$ \\
Fentanyl & $10.2 \%$ & $2.3 \%$ & .405 \\
Tramadol & $18.3 \%$ & $15.2 \%$ & .745 \\
Oxycodone & $\mathbf{1 5 . 5 \%}$ & $\mathbf{1 . 9 \%}$ & $\mathbf{. 0 1 6}$ \\
\hline
\end{tabular}

ASC, ambulatory surgery center; POD, postoperative day; SD, standard deviation.

${ }^{\mathrm{a} B}$ Boldface indicates statistical significance.

\section{Theoretical Analysis}

Statistical analysis of the theoretical OME consumption is detailed in Table 4. Figure 3 demonstrates that $69.7 \%$ of ASC patients consumed less than or equal to the 50 th percentile $(54.0 \mathrm{mg})$ of OMEs. In contrast, $64.3 \%$ of $\mathrm{HBC}$ patients consumed greater than $54.0 \mathrm{mg}$ OMEs. Of the total OME utilization, the ASC cohort consumed a greater dosage $(40.2 \pm 10.4$ versus $31.7 \pm 14.9, P$ $=.044)$ and percent of hydrocodone $(80.7 \%$ versus $55.7 \%, P=.014$; Figure 4) compared to HBCs. In contrast, patients at HBCs consumed a greater percent of oxycodone $(15.5 \%$ versus $1.9 \%, P=.016$; Figure 4) compared to ASCs.

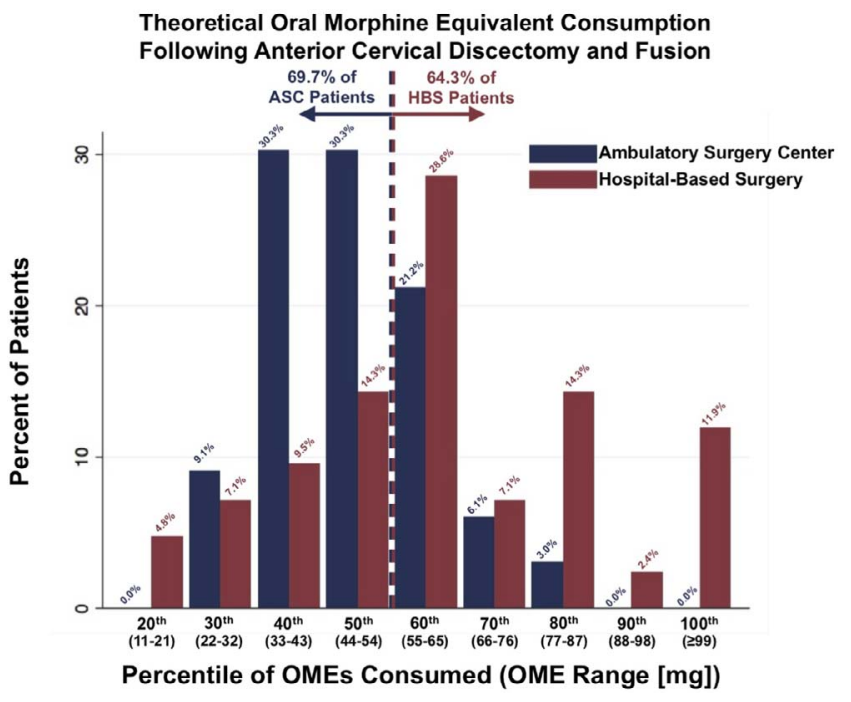

Figure 3. Theoretical oral morphine equivalent (OME) consumption following anterior cervical discectomy and fusion.

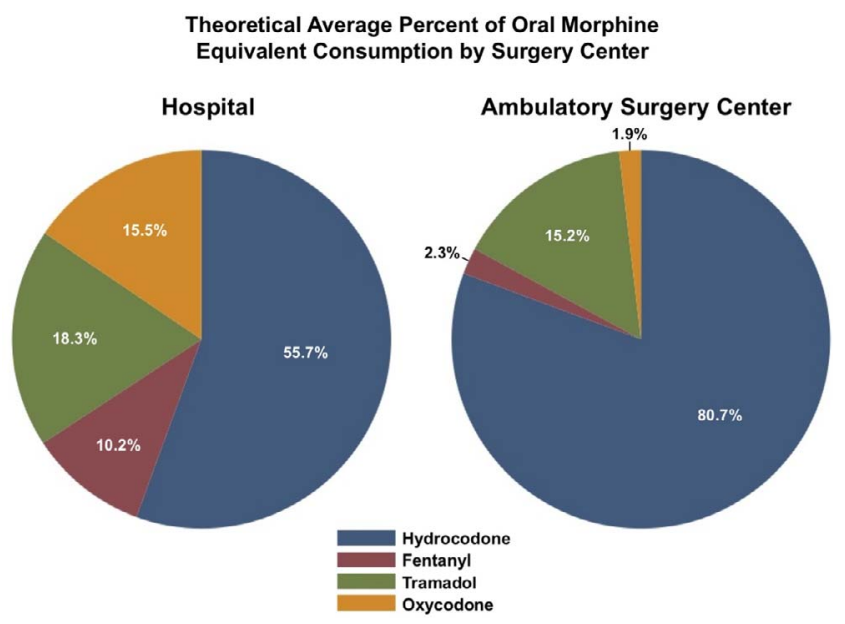

Figure 4. Theoretical average percent of oral morphine equivalent consumption by surgery center.

\section{DISCUSSION}

In the current health care climate, surgical practices have shifted toward performing procedures in outpatient facilities in order to maximize efficiency and minimize costs. ${ }^{19-29}$ Prior literature has reported the safety and efficacy of ACDF procedures in the outpatient and inpatient settings. ${ }^{7,9-15}$ Among other concerns, narcotic usage is associated with a significant side-effect profile, including nausea, vomiting, constipation, drowsiness, coughing, difficulty with deep breathing, transient impairment of psychomotor skills, and impaired bowel and bladder function. ${ }^{30-32}$ Concern for these side effects and the risk of opioid toxicity have led physicians to reduce the quantity of narcotics administered. ${ }^{30-33}$ In this context, the goal of the current study was to quantify narcotic consumption at an $\mathrm{HBC}$ versus an ASC for patients who underwent $\mathrm{ACDF}$ and were discharged on POD 0.

The results of the current study indicate that patients who underwent ACDF at an ASC consume significantly fewer total and hourly narcotics on POD 0 than patients at an HBC. Furthermore, the ASC and HBC cohorts achieved similar levels of pain prior to discharge despite the ASC cohort having a shorter LOS and receiving less total narcotic medication. Additionally, the $\mathrm{HBC}$ cohort consumed greater average dosages of all medications analyzed and also consumed increased amounts of the narcotic medications fentanyl and oxycodone. Relative to total OME consumption, the $\mathrm{HBC}$ cohort also consumed a greater percentage of oxycodone, whereas the ASC cohort consumed a 
greater percentage of the nonnarcotic medication tramadol.

As the HBC cohort experienced prolonged LOS compared to the ASC cohort, a theoretical analysis was performed in order to minimize the effect of LOS on narcotic consumption. The results of the theoretical analysis exhibited a trend toward greater narcotic consumption in the HBC cohort; however, this result was not statistically significant. The addition of theoretical postdischarge narcotic consumption also caused alterations in the patterns of narcotic consumption between cohorts. In this analysis, the ASC cohort consumed greater absolute amounts and percentages of hydrocodone compared to the HBC cohort. However, the HBC cohort still consumed greater amounts of all other medications, with differences in oxycodone and fentanyl reaching statistical significance. The trend of overall higher narcotic consumption of patients at HBCs, coupled with the fact that no significant differences existed in immediate postoperative pain, has potential implications for postoperative narcotic prescription patterns. These results suggest that surgeons may be able to reduce the quantity of inpatient narcotics administered in the early postoperative period to simulate a patient's outpatient narcotic regimen without compromising adequate pain control.

The findings of the current study are similar to those in the literature of other orthopaedic subspecialties. $^{34,35}$ With regard to patterns of analgesic utilization, Buchanan et $\mathrm{al}^{36}$ reported more effective pain control using the nonsteroidal anti-inflammatory drug diclofenac compared to the narcotic papaveretum following total hip replacement. Kao et $\mathrm{al}^{37}$ further demonstrated similar postoperative pain control between inpatient and outpatient surgical setting populations following ACL reconstruction, with the outpatient cohort consuming fewer narcotics. While several factors contribute to discharge planning following spinal surgery, the results of this and other studies suggest that a reduction in postoperative narcotic medications may result in shorter lengths of hospital stay and a subsequent reduction in health care costs.

This study also demonstrated no long-term differences in patient-reported outcomes, improvement, and complication rates between $\mathrm{HBC}$ and ASC cohorts. Previous literature has also demonstrated that ACDF procedures performed in the outpatient setting are at least noninferior to the traditional inpatient alternative. Single surgeon populations and national database studies report shorter operative times, similar or higher patient satisfaction ratings, similar or lower complication rates, lower morbidity rates, fewer returns to the operating room, and lower costs associated with ambulatory procedures. ${ }^{9-12,38-40}$ The equivalency of ambulatory ACDF to inpatient procedures with regard to long-term outcomes and complication rates, coupled with demonstrated advantages in operative time and costs within the literature, indicates that outpatient ACDF is a feasible, safe, and cost-effective treatment option.

While long-term outcomes were equivalent, patients who underwent their procedure at an ASC had higher VAS neck pain scores and reduced improvement in VAS neck pain at 6-week followup. This finding may be a result of the reduced narcotic dosing in the ASC cohort during the immediate postoperative period potentially leading to increased short-term pain after discharge. However, further study, including an analysis of postoperative narcotic usage past POD 0 , would be necessary to definitively make such a conclusion. Furthermore, as pain scores were not significantly different at 12-week and 6-month follow-up visits, the higher pain scores seen at the initial 6-week follow-up demonstrated no long-term effect on outcomes in the ASC population as compared to the HBC population.

The current study is not without limitations. First, the generalizability of this study is limited, as all procedures were performed by a single surgeon at single HBC and ASC sites. Second, the retrospective nature of this study may introduce selection bias with inherent differences between patients undergoing procedures at $\mathrm{HBCs}$ versus ASCs. However, the only differences in patient demographics or operative variables were in insurance status. Additionally, the risk of bias was also mitigated by the use of statistical analyses that were adjusted for all major preoperative demographic and baseline comorbidity characteristics. Third, the patient sample was small. As a result, this study may be underpowered to assess all differences in narcotic administration between surgical sites. This may have manifested in the loss of statistical significance on the theoretical analysis of narcotic consumption. Fourth, no measurement of preoperative narcotic use was available, and, as such, the effect of narcotic tolerance within the population could not be quantified. Finally, there was no direct measure- 
ment of postdischarge narcotics usage. While the theoretical analysis was used to estimate postdischarge narcotics utilization, it did not extend past POD 0. As such, longer-term narcotics utilization patterns that may have affected pain scores at follow-up intervals were not quantifiable.

\section{CONCLUSIONS}

In order to determine the safety and efficacy of outpatient spinal procedures, it is important to analyze differences in narcotic consumption and pain in the early postoperative period by surgical setting. The results of the current study suggest that patients at HBCs receive more potent narcotics in larger doses than patients at ASCs, which may contribute to the prolonged postoperative LOS. Although patients may be receiving more narcotics in HBCs, theoretical analysis may suggest that patients are receiving similar OMEs on POD 0 following $\mathrm{ACDF}$ regardless of operative location. Careful analysis of intraoperative anesthetic and postoperative medication protocols in an effort to minimize narcotic consumption in the postoperative period may reduce opioid toxicity, narcotic-related morbidity, LOS, and health care costs following ACDF.

\section{REFERENCES}

1. Martin BI, Deyo RA, Mirza SK, et al. Expenditures and health status among adults with back and neck problems. JAMA. 2008;299(6):656-664.

2. Martin BI, Turner JA, Mirza SK, Lee MJ, Comstock BA, Deyo RA. Trends in health care expenditures, utilization, and health status among US adults with spine problems, 1997-2006. Spine (Phila Pa 1976). 2009;34(19):2077-2084.

3. Andersson GB. Epidemiological features of chronic lowback pain. Lancet. 1999;354(9178):581-585.

4. Davis MA, Onega T, Weeks WB, Lurie JD. Where the United States spends its spine dollars: expenditures on different ambulatory services for the management of back and neck conditions. Spine (Phila Pa 1976). 2012;37(19):1693-1701.

5. Hoy DG, Protani M, De R, Buchbinder R. The epidemiology of neck pain. Best Pract Res Clin Rheumatol. 2010;24(6):783-792.

6. Ferrari R, Russell AS. Regional musculoskeletal conditions: neck pain. Best Pract Res Clin Rheumatol. 2003;17(1):5770.

7. Tomaras CR, Blacklock JB, Parker WD, Harper RL. Outpatient surgical treatment of cervical radiculopathy. $J$ Neurosurg. 1997;87(1):41-43.

8. Marawar S, Girardi FP, Sama AA, et al. National trends in anterior cervical fusion procedures. Spine (Phila Pa 1976). 2010;35(15):1454-1459.

9. Villavicencio AT, Pushchak E, Burneikiene S, Thramann
JJ. The safety of instrumented outpatient anterior cervical discectomy and fusion. Spine J. 2007;7(2):148-153.

10. Trahan J, Abramova MV, Richter EO, Steck JC. Feasibility of anterior cervical discectomy and fusion as an outpatient procedure. World Neurosurg. 2011;75(1):145-148; discussion 143-144.

11. Sheperd CS, Young WF. Instrumented outpatient anterior cervical discectomy and fusion: is it safe? Int Surg. 2012;97(1):86-89.

12. Samuel AM, Grauer JN, Rihn JA, Labrum JT. Twolevel anterior cervical discectomy and fusion: an outpatient surgery? J Spinal Disord Tech. 2015;28(10):349-351.

13. Lied B, Ronning PA, Halvorsen CM, Ekseth K, Helseth E. Outpatient anterior cervical discectomy and fusion for cervical disk disease: a prospective consecutive series of 96 patients. Acta Neurol Scand. 2013;127(1):31-37.

14. Baird EO, Egorova NN, McAnany SJ, Qureshi SA, Hecht AC, Cho SK. National trends in outpatient surgical treatment of degenerative cervical spine disease. Global Spine $J$. 2014;4(3):143-150.

15. Erickson M, Fites BS, Thieken MT, McGee AW. Outpatient anterior cervical discectomy and fusion. Am J Orthop (Belle Mead NJ). 2007;36(8):429-432.

16. Rodgers J, Cunningham K, Fitzgerald K, Finnerty E. Opioid consumption following outpatient upper extremity surgery. J Hand Surg Am. 2012;37(4):645-650.

17. Walid MS, Hyer L, Ajjan M, Barth AC, Robinson JS Jr. Prevalence of opioid dependence in spine surgery patients and correlation with length of stay. J Opioid Manag. 2007;3(3):127128, 130-132.

18. Hyer LA, Walid MS, Brooks AM, Darmohray DM, Robinson JS Jr. Interaction of age and opioid dependence on length of hospital stay for spine surgery patients. Psychol Rep. 2009;105(2):361-364.

19. Best NM, Sasso RC. Outpatient lumbar spine decompression in 233 patients 65 years of age or older. Spine (Phila $P a$ 1976). 2007;32(10):1135-1139; discussion 1140 .

20. Best NM, Sasso RC. Success and safety in outpatient microlumbar discectomy. J Spinal Disord Tech. 2006;19(5):334337.

21. Bookwalter JW III, Busch MD, Nicely D. Ambulatory surgery is safe and effective in radicular disc disease. Spine (Phila Pa 1976). 1994;19(5):526-530.

22. Gray DT, Deyo RA, Kreuter W, et al. Population-based trends in volumes and rates of ambulatory lumbar spine surgery. Spine (Phila Pa 1976). 2006;31(17):1957-1963; discussion 1964.

23. Helseth O, Lied B, Halvorsen CM, Ekseth K, Helseth E. Outpatient cervical and lumbar spine surgery is feasible and safe: a consecutive single center series of 1449 patients. Neurosurgery. 2015;76(6):728-737; discussion 737-728.

24. Lang SS, Chen HI, Koch MJ, et al. Development of an outpatient protocol for lumbar discectomy: our institutional experience. World Neurosurg. 2014;82(5):897-901.

25. Pugely AJ, Martin CT, Gao Y, Mendoza-Lattes SA. Outpatient surgery reduces short-term complications in lumbar discectomy: an analysis of 4310 patients from the ACS-NSQIP database. Spine (Phila Pa 1976). 2013;38(3):264-271.

26. Singhal A, Bernstein M. Outpatient lumbar microdiscectomy: a prospective study in 122 patients. Can J Neurol Sci. 2002;29(3):249-252. 
27. Techy F, Benzel EC. Implementing an outpatient ambulatory discectomy protocol at a large academic center: a change for the better. World Neurosurg. 2015;83(3):341-342.

28. Walid MS, Robinson JS III, Robinson ER, Brannick BB, Ajjan M, Robinson JS Jr. Comparison of outpatient and inpatient spine surgery patients with regards to obesity, comorbidities and readmission for infection. J Clin Neurosci. 2010;17(12):1497-1498.

29. Zahrawi F. Microlumbar discectomy. Is it safe as an outpatient procedure? Spine (Phila Pa 1976). 1994;19(9):10701074.

30. Mathiesen O, Dahl B, Thomsen BA, et al. A comprehensive multimodal pain treatment reduces opioid consumption after multilevel spine surgery. Eur Spine J. 2013;22(9):2089-2096.

31. Rajpal S, Gordon DB, Pellino TA, et al. Comparison of perioperative oral multimodal analgesia versus IV PCA for spine surgery. J Spinal Disord Techn. 2010;23(2):139-145.

32. Kehlet H, Dahl JB. Anaesthesia, surgery, and challenges in postoperative recovery. Lancet. 2003;362(9399):1921-1928.

33. Mahowald ML, Singh JA, Majeski P. Opioid use by patients in an orthopedics spine clinic. Arthritis Rheum. 2005;52(1):312-321.

34. Gimbel JS, Brugger A, Zhao W, Verburg KM, Geis GS. Efficacy and tolerability of celecoxib versus hydrocodone/ acetaminophen in the treatment of pain after ambulatory orthopedic surgery in adults. Clin Ther. 2001;23(2):228-241.

35. Post ZD, Restrepo C, Kahl LK, van de Leur T, Purtill JJ, Hozack WJ. A prospective evaluation of 2 different pain management protocols for total hip arthroplasty. J Arthroplasty. 2010;25(3):410-415.

36. Buchanan JM, Baldasera J, Poole PH, Halshaw J, Dallard JK. Postoperative pain relief; a new approach: narcotics compared with non-steroidal anti-inflammatory drugs. Ann R Coll Surg Engl. 1988;70(5):332-335.

37. Kao JT, Giangarra CE, Singer G, Martin S. A comparison of outpatient and inpatient anterior cruciate ligament reconstruction surgery. Arthroscopy. 1995;11(2):151156.

38. Liu JT, Briner RP, Friedman JA. Comparison of inpatient vs. outpatient anterior cervical discectomy and fusion: a retrospective case series. BMC Surg. 2009;9:3.

39. McGirt MJ, Godil SS, Asher AL, Parker SL, Devin CJ. Quality analysis of anterior cervical discectomy and fusion in the outpatient versus inpatient setting: analysis of 7288 patients from the NSQIP database. Neurosurg Focus. 2015;39(6):E9.

40. Adamson T, Godil SS, Mehrlich M, Mendenhall S, Asher AL, McGirt MJ. Anterior cervical discectomy and fusion in the outpatient ambulatory surgery setting compared with the inpatient hospital setting: analysis of 1000 consecutive cases. $J$ Neurosurg Spine. 2016;24(6):878-884.

Disclosures and COI: No funds were received in support of this work. No benefits in any form have been or will be received from any commercial party related directly or indirectly to the subject of this manuscript.

Corresponding Author: Kern Singh, MD, Professor, Department of Orthopaedic Surgery, Rush University Medical Center, 1611 West Harrison St, Suite 300, Chicago, IL 60612. Phone: (312) 432-2373; Fax: (708) 492-5373; Email: kern.singh@ rushortho.com.

Published 15 October 2018

This manuscript is generously published free of charge by ISASS, the International Society for the Advancement of Spine Surgery. Copyright (C) 2018 ISASS. To see more or order reprints or permissions, see http://ijssurgery.com. 